\title{
Can Mathematical Objects Be Causally Efficacious?
}

\begin{abstract}
Callard (2007) argues that it is metaphysically possible that a mathematical object, although abstract, causally affects the brain. I raise the following objections. First, a successful defence of mathematical realism requires not merely the metaphysical possibility but rather the actuality that a mathematical object affects the brain. Second, mathematical realists need to confront a set of three pertinent issues: why a mathematical object does not affect other concrete objects and other mathematical objects, what counts as a mathematical object, and how we can have knowledge about an unchanging object.
\end{abstract}

\section{Keywords}

Mathematical Causalism, Mathematical Object, Mathematical Realism

Seungbae Park

Ulsan National Institute of Science and Technology

Republic of Korea

nature@unist.ac.rk

Park, Seungbae (2018). "Can Mathematical Objects Be Causally Efficacious?" Inquiry: An Interdisciplinary Journal of Philosophy."

http://www.tandfonline.com/eprint/aj48ey2f9DjtErprQNk8/full

https://doi.org/10.1080/0020174X.2018.1432412

http://www.tandfonline.com/doi/full/10.1080/0020174X.2018.1432412

\section{Introduction}

According to mathematical realism, mathematical objects are inhabitants of the abstract world, so they are nonspatial, nontemporal, noncausal, and unchanging. An immediate objection to mathematical realism is that it is not clear how we acquire knowledge about them (Benacerraf 1973; Balaguer 2014). We are cognitive agents inhabiting the concrete world, and hence we cannot causally interact with them. We acquire knowledge about concrete objects only by physically interacting with them. But we cannot physically interact with mathematical objects. It follows that we cannot form knowledge about them.

This epistemological objection to mathematical realism elicited an original and intriguing response from Benjamin Callard (2007). He argues that though mathematical objects exist in the abstract world, they can nevertheless causally affect human brains and hence can produce mathematical knowledge. Let me call this view mathematical causalism. It is a view about how we can acquire mathematical knowledge under the mathematical realist framework. Mathematical realists might use causalism as a means to overcome Benacerraf's aforementioned epistemological objection. There are other means (Frege 1884; Gödel 1947) of which mathematical realists might avail themselves, but they fall outside the scope of this paper.

This paper aims to expose the limitations to Callard's causalist defence of mathematical realism. In Section 2, I demonstrate that a successful defence of mathematical realism requires not merely the metaphysical possibility but rather the actuality that a mathematical object affects the brain. In Section 3, I argue that causalists owe us two positive accounts of why a mathematical object affects the brain, but not other concrete objects and 
other mathematical objects. In Section 4, I claim that the causalist view that an object, even if causal, is mathematical opens a new debate over what counts as a mathematical object. In Section 5, I raise an objection to mathematical realism that we cannot have knowledge about a mathematical object because it cannot undergo any change.

\section{Metaphysical vs. Actual}

To say that a mathematical object can affect the human brain implies that the mathematical object can impart energy to the brain and receive energy from the brain. It is not clear, however, whether a mathematical object has such capability. Energy is spatial, and it exists in the concrete world. But how can the abstractum have an influence on the concretum?

Callard admits that it is metaphysically impossible for a mathematical object to receive energy from the brain, for 'any object receiving energy must change' $(2007,351)$. He argues, however, that it is metaphysically possible for a mathematical object to impart energy to the brain without itself suffering any change. He says, 'There is no contradiction, or any other conceptual or metaphysical difficulty, in accepting the claim that abstract objects impart energy to us, and thereby change us, without themselves receiving any energy or suffering any change' $(2007,351)$.

Newton's third law of motion, however, prevents an object from imparting energy to another object without receiving energy from it. So the law of action-reaction clashes with the causalist suggestion that a mathematical object can impart energy to the brain without receiving any energy from it. Callard replies that the third law of motion 'is just an empirical truth' $(2007,351)$; it remains metaphysically possible, although empirically or nomologically impossible, for a mathematical object to impart energy to the brain without receiving any energy from it. He says that 'efficient causal relations between non-spatial, necessary, eternal, unchanging objects and spatial, contingent, changing objects are strongly possible' (2007, 353). In other words, it is conceivable, metaphysically possible, or not self-contradictory for a mathematical object to affect the brain without itself undergoing any change.

Callard's foregoing defence indicates that he believes a mathematical object can impart energy to the brain, where 'can' means a metaphysical possibility, not an empirical or nomological possibility. But what does the metaphysical possibility prove? It proves nothing except that it is not self-contradictory that a mathematical object imparts energy to the brain. It is not clear how much the metaphysical possibility helps mathematical realism.

A successful defence of mathematical realism requires not merely the metaphysical possibility but rather the actuality that a mathematical object affects the brain. After all, it is not merely a metaphysical possibility but an actuality that we have mathematical knowledge, i.e., it is a plain fact that we have mathematical knowledge. Thus, the question for mathematical realists is not how it is metaphysically possible that we acquire mathematical knowledge but rather how we acquire mathematical knowledge. In other words, an explanandum for causalists is not the metaphysical possibility but the actuality.

In general, if an explanandum is an actuality, an explanans should also be an actuality, not merely a metaphysical possibility. Suppose, for example, that you are puzzled over why an apple falls down, and you ask me to provide an account of why it falls down. I say that an apple falls down because it is metaphysically possible that a gravitational force exists between the Earth and the apple. Such an account would hardly satisfy you. By contrast, you might be satisfied if I say that an apple falls down because a gravitational force exists between the Earth and the apple. This example illustrates that an actuality can be explained in terms of another actuality, but not in terms of a metaphysical possibility.

Benacerraf is puzzled not over how it is metaphysically possible that we have mathematical knowledge but over how we have mathematical knowledge, i.e., he is puzzled 
not over the metaphysical possibility but over the actuality. Callard, however, replies that we have mathematical knowledge because it is metaphysically possible for a mathematical object to affect the brain. He has provided the metaphysical possibility as an explanans for the actuality. Such an explanation would hardly satisfy Benacerraf. He would be satisfied if causalists provide the actuality that a mathematical object affects the brain, or at least if causalists provide an account of how neuroscientists can go about establishing the causal relationship between a mathematical object and the brain.

Causalists, however, cannot provide such an account. Suppose that a brain state correlated with mathematical knowledge occurs, and that neuroscientists are trying to investigate the cause of the brain state. Where should they look? Should they look inside the brain or outside the brain? When should they look? Should they look before or after the brain state occurs? It is meaningless even to ask such questions, given that a mathematical object is nonspatial and nontemporal. Thus, we return to Benacerraf's epistemological concern.

Callard might reply that a mathematical object, although far from the brain, can nonetheless affect it. After all, he claims that it is an intelligible notion that an object affects another object although they are far from each other. He says, "If "action at a distance"- that is, one physical object causally affecting another without (even indirectly) touching it - is intelligible, then the problem with the idea of causal interactions between ourselves and abstract objects is not to be found in the absence of impacts in the mathematical case (since these would be absent in cases of action at a distance too), but must be sought elsewhere' (2007, 350).

Causalists, however, cannot appeal to the notion of action at a distance in order to establish the causal relationship between a mathematical object and the brain. After all, to say that a mathematical object is distant from the brain implies that the mathematical object is spatial, which goes against the realist idea that it is nonspatial. Thus, it is a challenging task for causalists to specify how neuroscientists can locate the mathematical cause of a brain state.

\section{Other Concrete and Mathematical Objects}

Mathematical realists invoking causalism to defuse Benacerraf's epistemological objection to mathematical realism should confront the following two disconcerting issues.

First, can a mathematical object affect other concrete objects, such as rocks, water, and trees? There are far more other concrete objects than brains in the concrete world, so it is $a$ priori far more likely that a mathematical object affects other concrete objects than brains. How does a mathematical object affect a rock on a mountain? What mechanism does a mathematical object use to effect a change in a rock? Causalists might answer that a mathematical object, by nature, affects the human brain, but not other concrete objects. Such an answer, however, is ad hoc. Why is it that a mathematical object affects the brain, but not other concrete objects? What is so special about a mathematical object that enables it to have an influence on the brain, but not on other concrete objects? Does it give off a pheromone to which only the brain responds? What is so special about the brain that makes it sensitive to the influence of a mathematical object? Does it contain a gene that is attuned to the pheromone given off by a mathematical object? In short, causalists owe us an account of why a mathematical object affects the brain, but not other concrete objects.

Second, why is it that a mathematical object affects the brain, but not other mathematical objects? There are infinitely many mathematical objects, and they all inhabit the same world, viz., the abstract world. By contrast, there are finitely many brains, and they all inhabit a different world, viz., the concrete world. Therefore, it is a priori far more likely that a mathematical object affects other mathematical objects than that it affects the brain. Causalism, however, asserts that a mathematical object does not affect other mathematical 
objects, although it affects the brain. Such a position is ad hoc unless causalists provide an account of why a mathematical object affects the brain, but not other mathematical objects. Causalists cannot say that a mathematical object, by definition, cannot affect other mathematical objects, for such a semantic move would only invite an opposite semantic move: a mathematical object, by definition, cannot affect the brain.

\section{Tricle}

Mathematical realists utilizing causalism to solve Benacerraf's epistemological puzzle need to address another perplexing issue: What counts as a mathematical object?

As we have seen in the introduction of this paper, mathematical realism asserts that a mathematical object is an abstract object, and an abstract object is nonspatial, nontemporal, noncausal, and unchanging, i.e., that a mathematical object has the four properties of being nonspatial, nontemporal, noncausal, and unchanging. Causalism, however, suggests that an object can be mathematical even if it is causal, which implies that an object can be mathematical even if it loses one of the four properties. This new definition of a mathematical object, according to which a mathematical object is nonspatial, nontemporal, unchanging, but causal, opens the possibility that an object can be mathematical even if it loses two of the four properties. Hence, it invites another new definition of a mathematical object: a mathematical object is nonspatial and noncausal, but temporal and changing.

What would be an example of an object that fits this latest definition of a mathematical object? Imagine that there is an object that changes its shape from a triangle to a circle and back to a triangle with every second (Park 2017a, 102-103). We can call it a tricle. A tricle is nonspatial and noncausal, given that a triangle and a circle are nonspatial and noncausal. But it is temporal and changing, given that it changes its shape with the lapse of time.

Is a tricle a mathematical object or not? Causalists think that a mathematical object does not have to be noncausal. So one might think that a mathematical object does not have to be nontemporal and unchanging either. It would follow that a tricle is a mathematical object. A problem arises, however, if a tricle is a mathematical object. Where does it exist? It cannot exist in the abstract world, for it is not clear how many straight edges it has in the abstract world. Given that time does not pass in the abstract world, it is wrong to say that a tricle has three straight edges at some time intervals, but no straight edge at other time intervals. Causalists might argue that it exists neither in the concrete world nor in the abstract world but in a third world. The third world is inhabited by objects which are nonspatial and noncausal, but temporal and changing. This suggestion, however, is ad hoc. It posits the third world solely for the sake of diverting the argument from a tricle. There should be an independent reason for thinking that the third world exists.

Causalists might contend that a tricle is not a mathematical object because it is temporal and changing. This possible contention, however, invites an immediate objection. Why is it that an object can mathematical even if it is causal, but not if it is temporal and changing? Thus, Callard's suggestion that a mathematical object can be causally efficacious opens a whole new debate over what counts as a mathematical object. This new debate is so difficult to resolve that it is not clear whether it is advantageous or disadvantageous for mathematical realists to rely on causalism to solve Benacerraf's epistemological puzzle.

\section{Unchanging}

Mathematical realism is vulnerable to the following objection. To know about things is to act upon them, and if things are acted upon, they undergo changes. For example, to know about a cat is to act upon it. When we act upon a cat, it undergoes a change. But a mathematical object, by definition, cannot undergo any change. Therefore, we cannot know about it. 
Callard admits that we know about things as a result of their acting upon us, but insists that there is no reason for thinking that knowing 'essentially involves acting on the things known' (2007, 352, footnote). In other words, we can know about things, even if we do not act upon them.

Callard is right that we do not have to act upon things in order to know about them. For example, when we look at Jupiter in the night sky, we acquire knowledge about it, but we do not act upon it. However, a refutation of mathematical realism does not require that we should act upon things known. It only requires that the things known should undergo changes. Think about Jupiter again. It emits photons, some of which reach our eyes. As a result, we acquire knowledge about it. Emitting photons means undergoing changes. Without such changes, we cannot form knowledge about it. A mathematical object, however, cannot undergo any change. Therefore, we cannot form knowledge about it.

Callard's contention that a mathematical object does not suffer any change, although we form knowledge about it, indicates that he takes the property of unchanging to be an essential property of a mathematical object, i.e., he thinks that an object is not a mathematical object if it does not have the property of being unchanging. Recall, however, that he thinks that the property of being noncausal is an accidental property of a mathematical object, i.e., an object can be mathematical even if it does not have the property of being noncausal. Again, causalism opens the new issue of which properties of a mathematical object are essential and which properties of a mathematical object are accidental.

\section{Conclusion}

I have raised several objections to Callard's contention that it is metaphysically possible for a mathematical object to affect the brain. First, a successful defence of mathematical realism requires not merely the metaphysical possibility but rather the actuality that a mathematical object affects the brain. The prospect of establishing such actuality is dim, given that it is not clear where and when neuroscientists should look in order to establish the alleged causal connection between a mathematical object and the brain. Second, causalists need to confront the issue of why a mathematical object does not affect other concrete objects and other mathematical objects, the issue of what counts as a mathematical object, and the issue of how we can have knowledge about an unchanging object.

These objections constitute the prima facie reasons for thinking that mathematical realism is not tenable, and that we should seek an alternative position. There are alternative positions in the literature. For example, mathematical fictionalism is defended by Mark Balaguer (1996, 1998, 2001, 2009), Gideon Rosen (2001), and Mary Leng (2005a, 2005b, 2010). Mathematical inferentialism is defended by Seungbae Park (2017b). Neither mathematical fictionalism nor mathematical inferentialism asserts that $\mathrm{s}$ mathematical object is an abstractum, so none of them requires a causal connection between a mathematical object and the brain. I leave the task of examining these alternative positions to future occasions.

Acknowledgments: This paper improved a lot thanks to anonymous referees' useful comments. This work was supported by the Ministry of Education of the Republic of Korea and the National Research Foundation of Korea (NRF-2016S1A5A2A01022592).

\section{References}

Balaguer, M. 1996. Towards a nominalization of quantum mechanics. Mind 105 (418): 20926. 
Press.

1998. Platonism and anti-platonism in mathematics. Oxford: Oxford University

2001. A theory of mathematical correctness and mathematical truth. Pacific Philosophical Quarterly 82 (2): 87-114.

(2): $131-62$.

2009. Fictionalism, theft, and the story of mathematics. Philosophia Mathematica 17

2014. Platonism in metaphysics. The Stanford Encyclopedia of Philosophy. Edward

N. Zalta (ed.), URL $=\langle$ http://plato.stanford.edu/archives/spr2014/entries/platonism/>.

Benacerraf, P. 1973. Mathematical truth. The Journal of Philosophy 70 (19): 661-79.

Callard, B. 2007. The conceivability of Platonism. Philosophia Mathematica 15 (3): 347-56.

Frege, G. 1884. Die grundlagen der arithmetic: Eine logisch-mathematische untersuchung über den begriff der zahl. Breslau: Koebner.

Gödel, K. 1947. What is Cantor's continuum problem? The American Mathematical Monthly 54 (9): 515-25.

Leng, M. 2005a. Revolutionary fictionalism: A call to arms. Philosophia Mathematica 13 (3): 277-93.

2005b. Mathematical explanation. In Mathematical reasoning and heuristics. C. Cellucci and D. Gillies (eds.), London: King's College Publication, 167-189.

--------- 2010. Mathematics and reality. Oxford: Oxford University Press.

Park, S. 2017a. Two criticisms against mathematical realism. Diametros 52: 96-106.

--------- 2017b. In defense of mathematical Inferentialism. Analysis and Metaphysics 16: 7083.

Rosen, G. 2001. Nominalism, naturalism, epistemic relativism. Noûs 35 (s15): 60-91. 